\title{
Inhibition of prostate cancer growth using doxorubicin assisted by ultrasound-targeted nanobubble destruction
}

This article was published in the following Dove Press journal:

International Journal of Nanomedicine

29 July 2016

Number of times this article has been viewed

\author{
Xiaozhou Fan ${ }^{1, *}$ \\ Luofu Wang ${ }^{2, *}$ \\ Yanli Guo' \\ Xingyu Xiong' \\ Lianhua Zhu' \\ Kejing Fang'
}

'Department of Ultrasound, Southwest Hospital, ${ }^{2}$ Department of Urology, Daping Hospital,

Third Military Medical University, Chongqing, People's Republic of China

*These authors contributed equally to this work
Correspondence: Yanli Guo Department of Ultrasound, Southwest Hospital, 30 Gaotanyan Street, Shapingba, Chongqing 400038, People's Republic of China

Tel/fax +862368765439

Email guoyanli7ı@aliyun.com
Abstract: Ultrasound (US)-targeted microbubble destruction has been widely used as an effective drug-delivery system. However, nanobubbles (NBs) have better stability and stronger penetration than microbubbles, and drug delivery assisted by US-targeted NB destruction (UTND) still needs to be investigated. Our aim was to investigate the effect of doxorubicin (DOX) on the inhibition of prostate cancer growth under UTND. Contrast-enhanced US imaging of transplanted PC3 prostate cancer in mice showed that under a combination of $1 \mathrm{~W} / \mathrm{cm}^{2} \mathrm{US}$ power and a 100 $\mathrm{Hz}$ intermittent pulse with a " 5 seconds on, 5 seconds off" mode, NBs with an average size of $(485.7 \pm 33) \mathrm{nm}$ were effectively destroyed within 15 minutes in the tumor location. PC3 cells and 20 tumor-bearing mice were divided into four groups: a DOX group, a DOX + NB group, a DOX + US group, and a DOX + NB + US group. The cell growth-inhibition rate and DOX concentration of xenografts in the DOX + NB + US group were highest. Based on another control group and these four groups, another 25 tumor-bearing mice were used to observe the treatment effect of nine DOX injections under UTND. The xenografts in the DOX + NB + US group decreased more obviously and had more cellular apoptosis than other groups. Finally, electron microscopy was used to estimate the cavitation effect of NBs under US irradiation in the control group, NB group, US group, and NB + US group. The results of scanning electron microscopy showed that PC3 cells in the DOX + NB + US group had more holes and significantly increased cell-surface folds. Meanwhile, transmission electric microscopy confirmed that more lanthanum nitrate particles entered the parenchymal cells in xenografts in the NB + US group compared with the other groups. This study suggested that UTND technology could be an effective method to promote drugs to function in US-irradiated sites, and the underlying mechanism may be associated with a cavitation effect.

Keywords: ultrasound therapy, nanobubble, cavitation effect, prostate cancer

\section{Introduction}

Chemotherapy and gene therapy have become important means of tumor therapy. However, drugs/genes can only exert their therapeutic effects after reaching target tissues or target organs; otherwise, the side effects produced may be harmful to the human body. For the majority of chemotherapeutic drugs and gene drugs, identifying the proper drug/gene-delivery system to maintain high concentrations of the drug/gene in specific target tissues and exert the best therapeutic effects is difficult. Viral vectors, represented by adenoviruses and lentiviruses, have very high transduction efficiency; however, their potential infection and immunogenicity risks have not yet been effectively solved. Although liposome transfection plays an important role in in vitro cell studies, its transfection efficiency is low and its price is high. Therefore, their 
applications are just used in the experiments. Ultrasound (US)-targeted microbubble destruction (UTMD) technology can specifically destroy US contrast agents (UCAs) in USirradiated sites, and play an important role in the targeted delivery of drugs/genes. ${ }^{1,2}$ Currently, this technology is widely studied and explored in a variety of diseases, mainly cardiovascular diseases, eye diseases, and tumors. ${ }^{3-7}$

However, previous studies in this field focused mainly on micron-level UCAs; there are few studies on the recently developed nanoscale UCAs. Nanoscale UCAs are contrast agents with a particle diameter $<1,000 \mathrm{~nm}$, and can be used for imaging under contrast-enhanced US (CEUS) mode. Compared to microscale UCAs, nanoscale UCAs have features of strong penetration ability and excellent stability; therefore, they have been studied extensively, especially in applications in solid-tumor imaging. ${ }^{8,9}$ Nanoscale UCAs can be distributed more extensively in tumor tissues using enhanced permeation and retention (EPR) effects: large vascular endothelial gaps, incomplete basement membranes, and imperfect lymphatic drainage. ${ }^{10}$ In our previous studies, we constructed nanobubbles (NBs) carrying small interfering RNA against the androgen receptor. The NBs that carried androgen-receptor small interfering RNA were destroyed by US irradiation, and experiments on in vitro prostate cancer cells and xenograft tumors in animals were done. The results showed that this technique had good inhibitory effects on tumors. ${ }^{11,12}$ However, chemotherapeutic drugs assisted by US-targeted NB destruction (UTND) for the treatment of tumors and the underlying mechanism still require further investigation. Therefore, we applied lipid NBs and the classic broad-spectrum chemotherapeutic drug doxorubicin (DOX) to treat prostate cancer using UTND in this study. Next, we further studied the underlying mechanism using electron microscopy in order to provide a research basis for the inhibition of tumor growth by chemotherapeutic drugs using UTND.

\section{Materials and methods Preparation of ultrasound NBs}

The lipid components 1,2-dipalmitoyl-sn-glycero-3phosphocholine (DPPC; Avanti Polar Lipids, Alabaster, AL, USA), 1,2-dipalmitoyl-sn-glycero-3-phosphatidic acid (DPPA; Corden Pharma, Liestal, Switzerland), 1,2-dipalmitoyl-sn-glycero-3-phosphoethanolamine (DPPE; Corden Pharma), 1,2-dipalmitoyl-sn-glycero-3phosphoglycerol (DPPG; Corden Pharma), and polyethylene glycol 2000, were dissolved in a hydration solution containing glycerin and phosphate-buffered saline (PBS) at a ratio of 1:9 (v:v) and incubated overnight in a horizontal shaker at $37^{\circ} \mathrm{C}$.
The solution was transferred to a penicillin bottle, the air in the head of the bottle replaced with perfluoropropane gas $\left(\mathrm{C}_{3} \mathrm{~F}_{8}\right.$; Research Institute of Physical and Chemical Engineering of Nuclear Industry, Tianjin, People's Republic of China), and the bottle shaken using the horizontal reciprocating mechanical oscillation method with an operating frequency greater than 4,500 rpm for 80 seconds using an ST-B series amalgamator (AT\&M Biomaterials Co, Beijing, People's Republic of China). The obtained suspension was centrifuged sequentially under conditions of $300 \times g$ and $300 \mathrm{rpm}$ for 3 minutes to discard the lipid materials that were not encapsulated into NBs and microbubbles in the top layer.

After the separated NBs were negatively stained with a phosphotungstic acid solution, the size and morphology of the NBs were observed using transmission electron microscopy (TEM; JEM-1400; JEOL, Tokyo, Japan) and under the bright field of laser-scanning confocal microscopy (Zeiss 780; Carl Zeiss AG, Oberkochen, Germany), respectively. The particle sizes of the fabricated NBs were measured in a Malvern Zetasizer Nano ZS90 detector (Malvern Instruments, Malvern, UK) according to the principles of dynamic light scattering after they had been diluted with PBS.

\section{In vitro cell culture and the establishment of the tumor-xenograft animal model}

The PC3 prostate cancer cell line from the American Type Culture Collection was cultured in 1640 culture medium containing $10 \%$ fetal bovine serum and $1 \%$ penicillin and streptomycin in a $5 \% \mathrm{CO}_{2}$ and $37^{\circ} \mathrm{C}$ incubator. During the establishment of tumor xenografts into 4- to 5-week-old BALB/c-nu nude mice (HFK Bioscience Co, Beijing, People's Republic of China), $200 \mu \mathrm{L}$ of tumor cells in logarithmic phase at a concentration of $2.5 \times 10^{7} / \mathrm{mL}$ were mixed with $200 \mu \mathrm{L}$ of Matrigel (BD Biosciences, San Jose, CA, USA) and subcutaneously injected into nude mice. The mice were continuously housed in a clean environment. The animal experiments in this study were approved by the Institutional Animal Ethics Committee of the Third Military Medical University, and performed in accordance with the International Guiding Principles for Biomedical Research involving Animals-1985.

\section{In vitro cytotoxicity}

In vitro cytotoxic activity of DOX (Solarbio, Beijing, People's Republic of China) against PC 3 cells was evaluated by using the Cell Counting Kit (CCK)-8 assay (Dojindo Laboratories, Kumamoto, Japan). Briefly, cells $\left(5 \times 10^{3}\right.$ cells/well $)$ were seeded into 96-well plates and cultured for 12 hours to adhere at the bottom of the plates. Then, different concentrations 
of DOX, 0-50 $\mu \mathrm{M}$, were added and further incubated for 8 hours. After that, the medium was changed and replaced with fresh medium containing CCK-8 solution, and cells were further incubated for 1 hour. Finally, the absorbance of the sample taken from each well was measured at $450 \mathrm{~nm}$ in a Varioskan Flash multimode reader (Thermo Fisher Scientific, Waltham, MA, USA), on the basis of which the percentage of surviving cells at every concentration was calculated relative to the untreated one. After the optimal concentration of DOX against PC3 cells had been determined, the cells were divided into four groups: the DOX-only group, the DOX combined with NB (DOX + NB) group, DOX under US irradiation (DOX + US) group, and DOX combined with NB under US irradiation (DOX + NB + US) group. In the relevant groups, the intervention was done according to the following settings: the optimal dose of DOX as aforementioned $(10 \mu \mathrm{M}$ DOX), NBs $5 \times 10^{6}$, US power $1 \mathrm{~W} / \mathrm{cm}^{2}$, and irradiation time 20 seconds in WED-100 US therapeutic equipment (Well D Medical Electronics Co Ltd, Shenzhen, People's Republic of China). A similar procedure with CCK-8 assay was used to calculate cell viability in each treatment group. All experiments were performed in triplicate.

\section{Measurement of DOX concentrations in all tissues and organs of nude mice}

First, US power and time required for US burst were confirmed in nude mice. Injection of $5 \times 10^{7} \mathrm{NBs}$ was performed through the retro-orbital venous sinus of tumor-bearing mice. The WED-100 US therapeutic equipment was used to irradiate tumor regions from the side of tumor xenografts. A sufficient amount of coupling agent to fill the region between the probe and the tumor xenografts was used. The frequency of the therapeutic probe was $1 \mathrm{MHz}$. Irradiation of tumor regions was performed using US power of $1,1.75$, and $2.5 \mathrm{~W} / \mathrm{cm}^{2}$. To ensure reperfusion of the tumor regions after NB destruction, the built-in $100 \mathrm{~Hz}$ pulse of the therapeutic equipment combined with the manual " 5 seconds on, 5 seconds off" mode was used. During the process of US irradiation, an L12-5 probe scanning from the upper part of xenografts was initiated in superficial CEUS mode of an iU22 US diagnostic apparatus (Philips, Amsterdam, the Netherlands), which is used for real-time monitoring of the perfusion of NBs in tumor regions under the condition of 0.12 mechanic index until no NBs were observed inside the tumor xenografts. This period of time was considered the duration of US irradiation of NBs.

Next, $50 \mu \mathrm{g} / \mathrm{mL}$ DOX was prepared using double-distilled water, and whole-spectrum absorbance was measured at
200-1,000 $\mathrm{nm}$ in a multimode reader. After the optimal measuring wavelength was determined, standard curves of DOX at a concentration range of $1-50 \mu \mathrm{g} / \mathrm{mL}$ were generated. A total of 20 tumor-bearing nude mice with tumor sizes of $\sim 1 \mathrm{~cm}$ were randomly divided into four groups as described in the "In vitro cytotoxicity" section. The intervention was performed according to the following settings: injection dose of DOX $0.1 \mathrm{mg}$, NBs $5 \times 10^{7}$, and therapeutic US condition performed in tumor-xenograft regions using the aforementioned irradiation parameters.

Finally, the nude mice were killed 6 hours after the end of the experimental treatment. Livers, implanted tumors, kidneys, hearts, lungs, and spleens of the nude mice of each group were collected and fully minced for US tissue homogenization. DOX was extracted from the tissue supernatant using chloroformmethanol extraction. Supernatants of these tissues were collected from normal tumor-bearing mice and added to a series of DOX with known concentrations for the same manipulation. Absorbance was measured, and standard curves were generated to calculate the drug concentrations in all the organs.

\section{Treatment for tumor xenografts in nude mice}

Another 25 nude mice were randomly grouped, with five animals in each group. On the basis of the previous four groups, a blank control group was added. Two CEUS images of tumors were examined with a dosage of $1 \times 10^{7} \mathrm{NBs} 2$ days before treatment and 2 days after the end of treatment. The maximum intensities of signals were extracted using QLab8.1 offline software (Philips) for comparison. The specific treatment procedures were strictly carried out as described in the following content. When the diameter of the tumor xenograft was $5 \mathrm{~mm}$, treatment was initiated. The treatment was performed nine times every other day. The US parameters in the corresponding groups were the same as the above. The amount of injected DOX was $0.05 \mathrm{mg}$, and the amount of injected NBs was $5 \times 10^{7}$. The tumor volume and the mass of the nude mice on treatment days $0,6,12$, and 18 were recorded. The tumor volume was calculated as $\mathrm{V}=\pi / 6 \times \mathrm{L} \times \mathrm{W} \times \mathrm{H}$. Mice were killed by cervical dislocation 2 days after the last treatment. Tumor tissues and myocardial tissues were collected. The tumor xenografts were weighed, and tumor density was calculated as $\rho=\mathrm{M} / \mathrm{V}$.

\section{Assessment of tumor-xenograft and myocardial tissue sections}

Heart and tumor xenografts of the nude mice in the five groups were washed, fixed in $4 \%$ paraformaldehyde, embedded in 
paraffin, sectioned, and stained with hematoxylin-eosin (H\&E). In addition, the apoptosis conditions of cells in myocardial tissue and tumor-xenograft sections were analyzed according to the instruction manual of the terminal deoxynucleotidyl transferase deoxyuridine triphosphate nick-end labeling (TUNEL) assay reagent kit (Beyotime, Shanghai, People's Republic of China). According to the principle of the TUNEL method, terminal deoxynucleotidyl transferase functions to transfer peroxidase-modified deoxyribonucleotides to the 3'-OH terminus of DNA broken during cell apoptosis, allowing apoptosis to be observed in situ.

\section{Analysis of biological effects under UTND using electron microscopy}

First, the effects of NBs on surface morphology under US irradiation were observed at the cell level. PC3 prostate cancer cells $\left(1 \times 10^{4}\right)$ were inoculated onto 24 -well plates. The cells were cultured overnight to allow them to attach to the surface of coverslips. The cells were washed with PBS and divided into four groups: control, NB, US, and NB + US. The number of NBs in each well was $1 \times 10^{7}$. The wells were filled with PBS, and irradiation was performed for 20 seconds from the top of cell wells using the aforementioned US therapeutic probe. Next, the cells were rapidly washed with PBS, fixed in $2.5 \%$ glutaraldehyde for 2 hours, and dehydrated for replacement using different gradients of alcohol and tert-butyl alcohol. Samples were placed in a sample holder for 30 minutes at $4{ }^{\circ} \mathrm{C}$ and were vacuumed for spraying treatment. Finally, the samples were placed under scanning electron microscopy (SEM; S-3400N II; Hitachi Ltd, Tokyo, Japan) to observe the condition of the cell surface.

The effects of NBs under US irradiation on tumor xenografts were observed in tumor-bearing mice. A total of 20 tumor-bearing mice were divided into the following four groups: control, NB, US, and NB + US. Mice in all the groups were slowly injected with $0.1 \mathrm{~mL}$ of lanthanum nitrate solution with a mass fraction of $2 \%$ (prepared in sodium cacodylate) via the retro-orbital venous sinus. The US parameters and the amount of NBs were the same as those in the treatment procedure. After that, the nude mice were killed, and the tumor xenografts were rapidly collected, fixed in $2.5 \%$ glutaraldehyde at $4^{\circ} \mathrm{C}$ for 24 hours, and washed. Tissue blocks were fixed in $1 \%$ osmium tetroxide for 2 hours, dehydrated in an acetone gradient, and embedded in epoxy 168. Ultrathin tissue sections were prepared. Each group was divided into electron staining and non-electron-staining conditions. Electron staining was performed using uranyl acetate and lead nitrate. Finally, the distribution of lanthanum nitrate in tumor-xenograft tissues was observed under TEM.

\section{Statistical analysis}

Histograms and line charts were generated using GraphPad Prism 6.0 (GraphPad Software, La Jolla, CA, USA). Statistical analysis was performed using SPSS 22.0 software (IBM Corporation, Armonk, NY, USA). Data are presented as mean \pm standard deviation. The drug concentrations in all the organs and the data derived from evaluating the treatment in all the groups were analyzed using a one-way analysis of variance. $P<0.05$ indicated that a difference had statistical significance.

\section{Results}

\section{In vitro observation of NBs}

The prepared NBs appeared to be in milky suspension. After centrifugation and stratification treatment, the obtained NBs exhibited a dark-black round region encapsulated in a light-black circular structure with homogeneous size distribution under TEM (Figure 1A). Under the bright field of laser-scanning confocal microscopy, NBs were evenly distributed and showed a round shell structure (Figure 1B). The particle size of the obtained NBs was $485.7 \pm 33 \mathrm{~nm}$, and the polydispersity index was 0.026 according to a Malvern detector (Malvern Instruments; Figure 1C). The distribution range of the particle size was narrow, which was conducive to single-frequency US excitation.

\section{In vitro cell viability under UTND}

We first demonstrated in escalating-dose studies of DOX $(0-50 \mu \mathrm{M})$ that the cytotoxicity of DOX reached a plateau after $10 \mu \mathrm{M}$ of DOX had been added to PC 3 cells. Through CCK-8 assay, cell viability was $43.16 \% \pm 2.47 \%$ in the DOX + $\mathrm{NB}+$ US group, $54.15 \% \pm 1.52 \%$ in the DOX + US group, $72.47 \% \pm 1.7 \%$ in the DOX $+\mathrm{NB}$ group, and $71.9 \% \pm 2.32 \%$ in the DOX group (Figure 2). Except for the fact that there was no statistical difference between the DOX + NB group and the DOX group, the differences in cell viabilities between any two groups were significant $(P<0.05)$. The results indicated that US had a synergetic inhibition effect in DOX against PC3 cells, and NBs could strengthen this inhibition effect under US irradiation.

\section{Distribution conditions of DOX in different groups}

US monitoring with CEUS mode showed that NBs could be completely destroyed under three different levels of US power $\left(1,1.75\right.$, and $\left.2.5 \mathrm{~W} / \mathrm{cm}^{2}\right)$ (Figure 3). However, the next pulse had already been emitted after the burst of NBs by the previous pulse in the built-in $100 \mathrm{~Hz}$ pulse, and thus NBs could not perfuse the xenograft region fully. Therefore, an effective 

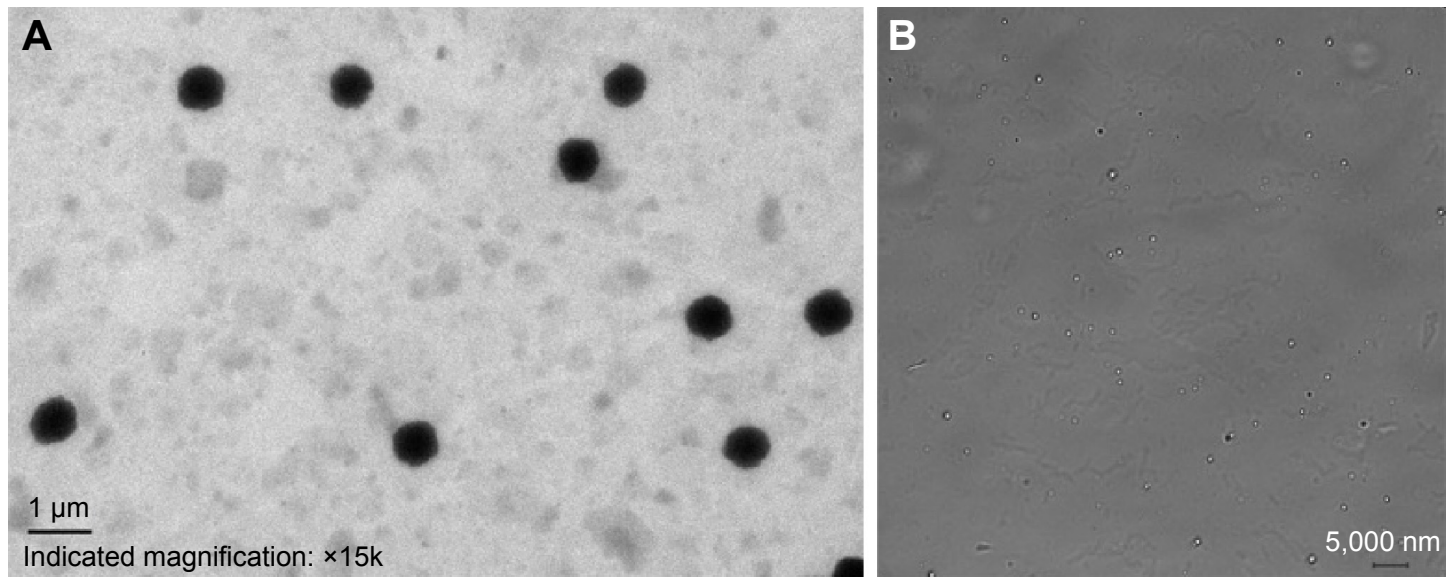

\begin{tabular}{lllll}
\hline & & Size (4 nm): & \% intensity: & SD (4 nm): \\
\cline { 2 - 5 } Z-average (4 nm): 445.0 & Peak 1: & 485.7 & 100 & 65.9 \\
Pdl: 0.026 & Peak 2: & 0 & 0 & 0 \\
Intercape: 0.842 & Peak 3: & 0 & 0 & 0 \\
Result quality: refer to quality report & & & \\
\hline
\end{tabular}

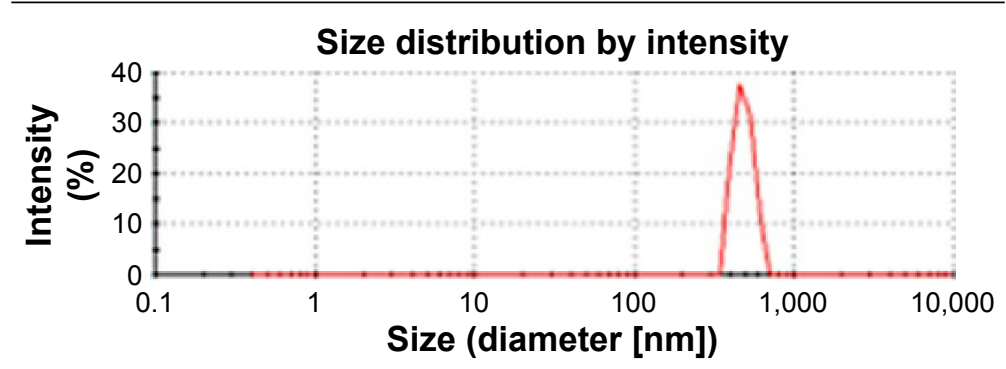

Figure I Morphology and size distribution of NBs.

Notes: (A) NBs observed under TEM after negative staining; (B) NBs observed under LSCM; (C) size-distribution curve of NBs from dynamic light-scattering test in a Malvern detector.

Abbreviations: LSCM, laser-scanning confocal microscopy; NBs, nanobubbles; SD, standard deviation; TEM, transmission electron microscopy; PdI, polydispersity index.

burst of NBs could not be achieved in the xenograft region, and the biological effects of the NBs could not be effectively exerted in the xenograft region. Therefore, for selection of the US irradiation mode, the built-in $100 \mathrm{~Hz}$ in the US therapeutic

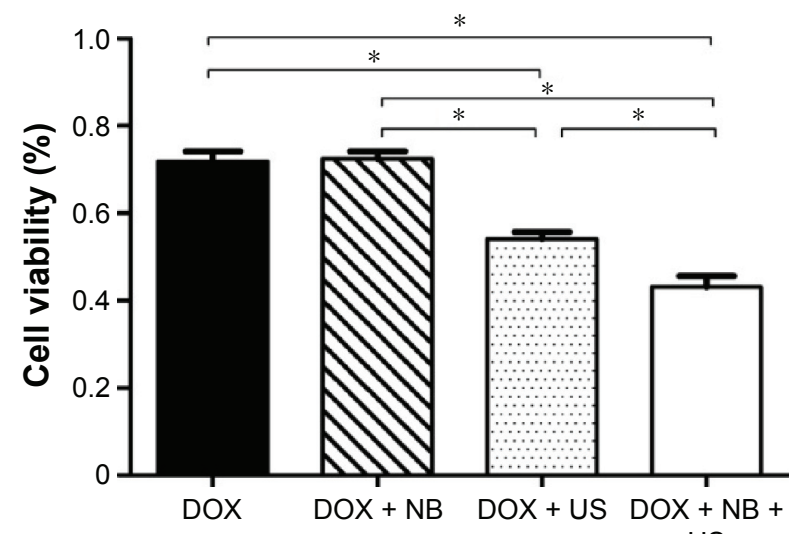

US

Figure 2 Growth-inhibition assay (CCK-8) for PC3 cells in different treatment groups. Note: $* p<0.05$.

Abbreviations: DOX, doxorubicin; NB, nanobubble; US, ultrasound. equipment combined with the manual functional 5 seconds on-5 seconds off mode was used. For the selection of US power, it was shown that the US therapeutic probe became significantly hot after a certain period of high-US power input. Therefore, we selected the lowest US power in this equipment, $1 \mathrm{~W} / \mathrm{cm}^{2}$, for irradiation to exclude the interference of thermal effects produced by US. Real-time observation using the US diagnostic apparatus showed that NBs in the circulation of the body of mice could be completely destroyed within 15 minutes under those conditions of therapeutic US in the xenograft region.

DOX absorbance was measured using full-spectrum scanning. The results showed that DOX absorbance had two peaks: 227 and $485 \mathrm{~nm}$. Different concentrations of DOX were used to generate curves. The correlation analysis between absorbance and DOX concentrations in the supernatant of all the relevant tissues and organs had the best linear correlation at the $485 \mathrm{~nm}$ wavelength (Table 1). Through the measurement of DOX absorbance in the supernatants of 


\section{A}

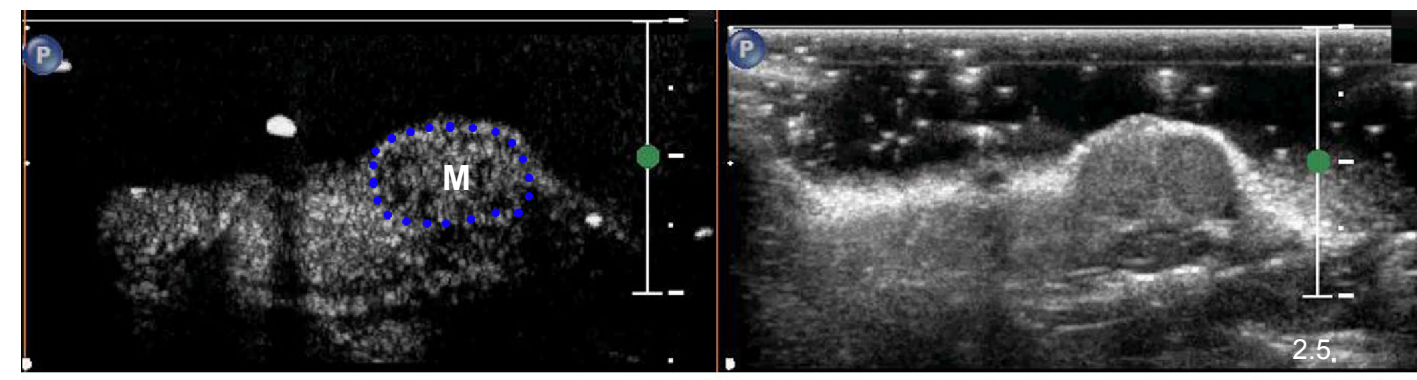

B

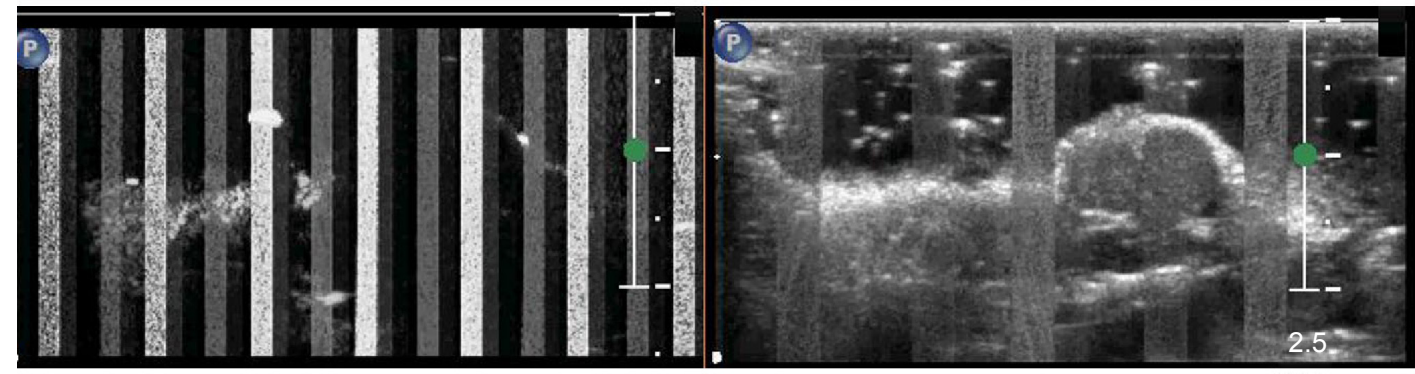

C

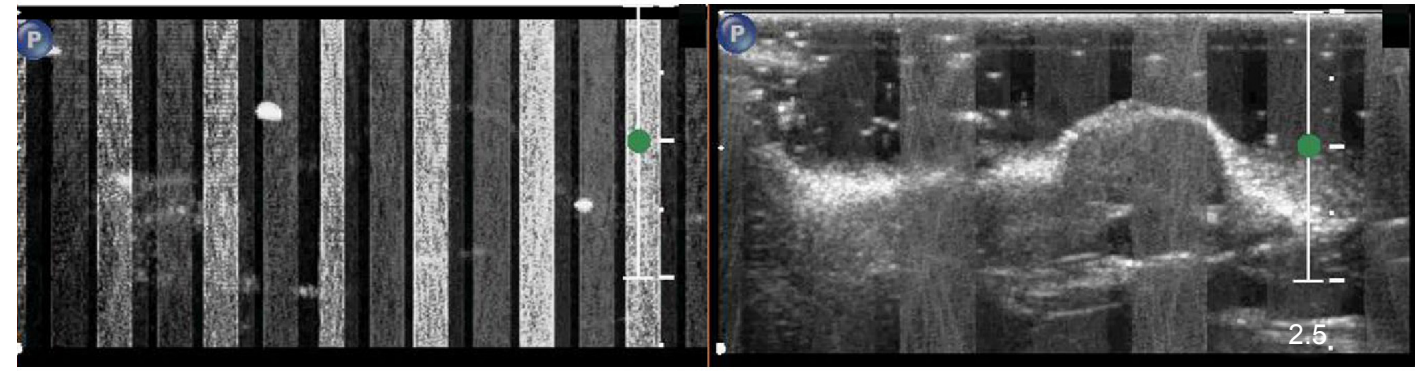

D

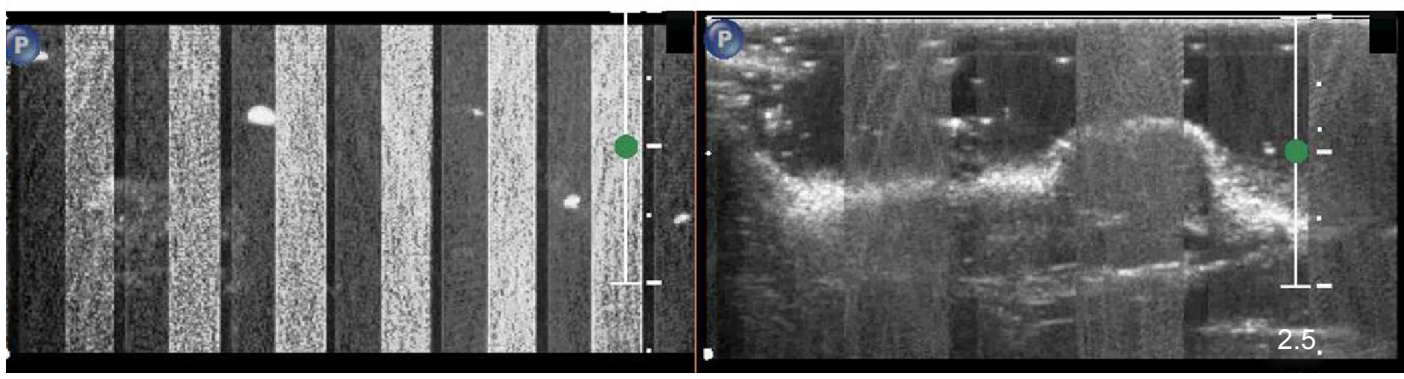

Figure 3 Destruction of NBs in tumor xenografts at different ultrasonic power intensities.

Notes: (A) Imaging of NBs in tumor xenografts under conventional contrast-enhanced grayscale ultrasound. (B-D) Destruction and waveform conditions of NBs in tumorxenograft regions under three intensities of ultrasonic power: I, I.75, $2.5 \mathrm{~W} / \mathrm{cm}^{2}$, respectively.

Abbreviations: NBs, nanobubbles; M, mass.

Table I Standard curves between the absorbance and the DOX concentration in different tissue supernatants

\begin{tabular}{lll}
\hline Solution & $\boldsymbol{R}^{2}$ & Formula \\
\hline Methanol & 0.992 & $y=0.002 x+0.046$ \\
Liver homogenate & 0.981 & $y=0.002 x+0.056$ \\
Spleen homogenate & 0.986 & $y=0.002 x+0.053$ \\
Kidney homogenate & 0.991 & $y=0.002 x+0.061$ \\
Pulmonary homogenate & 0.982 & $y=0.003 x+0.05$ \\
Myocardial homogenate & 0.981 & $y=0.002 x+0.06$ \\
Tumor homogenate & 0.998 & $y=0.003 x+0.053$ \\
\hline
\end{tabular}

Note: Absorbance at $485 \mathrm{~nm}$ indicated by $y$; DOX concentration $(\mu \mathrm{g} / \mathrm{mL})$ indicated by $x$.

Abbreviation: DOX, doxorubicin. livers, tumor xenografts, kidneys, hearts, lungs, and spleens of nude mice in all the groups, DOX concentrations in these tissues were obtained (Figure 4).

After statistical analysis, there were no significant differences in any two DOX concentrations from kidneys, lungs, and spleens in the four groups. However, DOX concentrations from any two groups in livers, tumors and hearts showed a significant difference $(P<0.05)$, except between the DOX group and the DOX + NB group. In detail, DOX concentrations in tumor-xenograft tissues were $43.71 \pm 5.03 \mathrm{ng} / \mathrm{g}$ in the DOX + NB + US group, $36.9 \pm 5.72 \mathrm{ng} / \mathrm{g}$ in the 


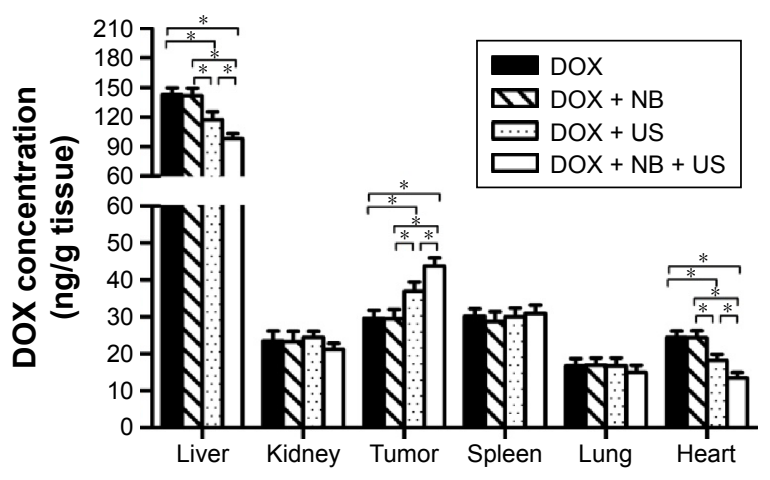

Figure 4 Distribution of DOX in a variety of tissues in the four groups. Note: $* P<0.05$.

Abbreviations: DOX, doxorubicin; NB, nanobubble; US, ultrasound.

DOX + US group, $29.48 \pm 5.76 \mathrm{ng} / \mathrm{g}$ in the DOX + NB group, and $29.58 \pm 4.82 \mathrm{ng} / \mathrm{g}$ in the DOX group. However, DOX concentrations in myocardial tissues and hepatic tissues in all groups generally showed the opposite distribution, in contrast with tumor tissues: the concentrations from myocardial tissues and hepatic tissues in the DOX group and DOX $+\mathrm{NB}$ groups were the highest, followed by the DOX + US group (18.21 \pm 3.63 and $117.22 \pm 18.14 \mathrm{ng} / \mathrm{g}$, respectively), and DOX concentrations in the DOX + NB + US group were the lowest (13.49 \pm 3.14 and $97.92 \pm 11.25 \mathrm{ng} / \mathrm{g}$, respectively). All of these results suggested that US irradiation in the tumor region could shift the DOX distribution from some specific organs to the irradiation tumor, and NBs under US irradiation could also enhance the effect. It was beyond the scope of our study as to why no differences of DOX concentrations in the four treatment groups occurred in the kidney, lung, or spleen.

\section{Effects of DOX on the inhibition of xenograft growth under UTND technology}

According to the experimental schedule (Figure 5A), five groups of mice were treated, and some indicators were observed. The results showed that the xenograft volumes, the body weights of mice, and reductions in peak intensity in CEUS mode in the DOX + NB + US group and DOX + US group were significantly different from those in the control group $(P<0.05$; Figure $5 \mathrm{~B}-\mathrm{G})$. Tumor volumes in the $\mathrm{DOX}+\mathrm{NB}+\mathrm{US}$ group $\left(68.16 \pm 14 \mathrm{~mm}^{3}\right)$ were smaller than those in the DOX + US group $\left(150.87 \pm 48.35 \mathrm{~mm}^{3}, P<0.05\right)$, while the body weight of mice and peak intensity of US imaging between these two groups showed no significant difference $(23.44 \pm 0.48 \mathrm{~g}$ vs $23.48 \pm 0.76 \mathrm{~g}, 14.2 \pm 1.4 \mathrm{~dB}$ vs $15.5 \pm 0.84 \mathrm{~dB} ; P>0.05)$. US imaging under CEUS mode in the DOX $+\mathrm{NB}+$ US group also showed an unfilled echo-free region in the center of the tumor xenografts, indicating necrosis inside the tumor xenografts, and the DOX + US group had the second-highest level of necrosis (Figure 5D). Xenograft density was calculated according to the mass and volume of the xenograft. The results showed that density in the DOX + NB + US group $\left(1.06 \pm 0.03 \mathrm{~g} / \mathrm{cm}^{3}\right)$ and the DOX + US group $\left(1.16 \pm 0.03 \mathrm{~g} / \mathrm{cm}^{3}\right)$ was significantly decreased compared to that of the control group $\left(1.49 \pm 0.13 \mathrm{~g} / \mathrm{cm}^{3}\right)$, while xenograft masses from any two groups were significantly different, except between the DOX and DOX + NB groups (Figure 5H). Through these analyses, the volume and mass of xenografts were sensitive indicators in reflecting the synergetic effect of NBs on DOX against the implanted tumor under US irradiation, but the density of tumor and the body weight of mice were not.

Myocardial tissue sections from animals in the five groups showed that there were no obvious differences in the H\&E sections. The results of TUNEL staining, reflecting apoptosis conditions, indicated that some cardiomyocytes underwent apoptosis in the DOX and DOX + NB groups (Figure 6A-J). The H\&E staining results of xenograft tissue sections revealed obvious cellular necrosis in the DOX + US and DOX + NB + US groups, which was also confirmed by TUNEL staining (Figure 6K-T). The overall results of the myocardial tissue and xenograft sections showed a negative correlation.

\section{Observations about effects of UTND on cells and tumor xenografts with electron microscopy}

To investigate whether NBs could produce cavitation effects under US irradiation, we designed four groups: control, NB, US, and NB + US. Patterns of changes in prostate cancer cells and tumor xenografts were observed using SEM and TEM, respectively. SEM results showed that the cell surface had significant changes in the NB + US group; there were more folds and small holes. Although cells in the US group had small holes, the number of holes was significantly reduced. The surfaces of the PC3 cells were smooth, and the holes on the surface were not obvious in the NB or control groups (Figure 7A-D). TEM results showed that the microstructure of all the groups had limited changes after the prostate cancer xenografts were stained with uranyl acetate and lead nitrate for electron staining. However, dense particles formed by lanthanum could be clearly observed inside cells in the US and NB + US groups (Figure 7E-H). To confirm further the presence of lanthanum agents in cells and tissues, the 

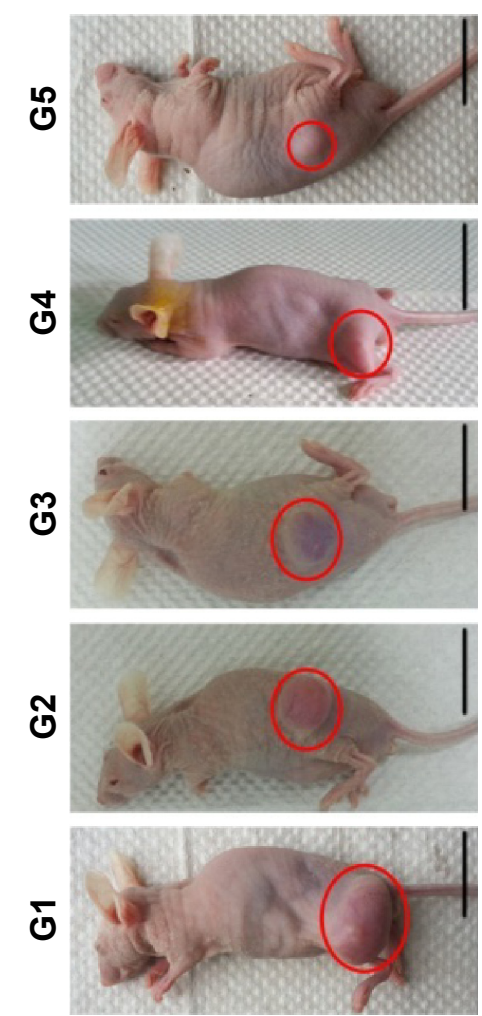

$\mathbf{m}$

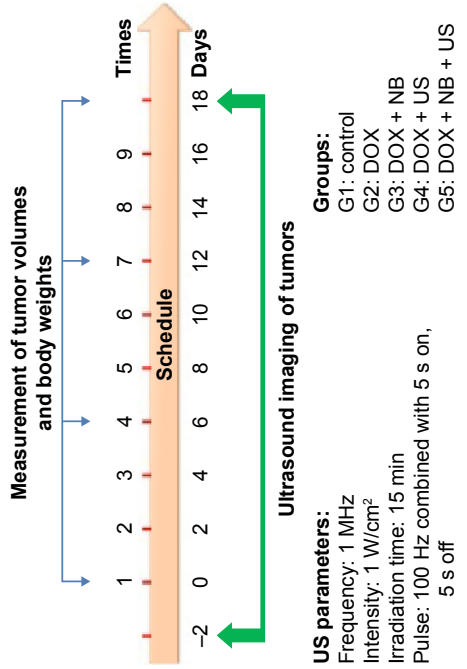

$\varangle$

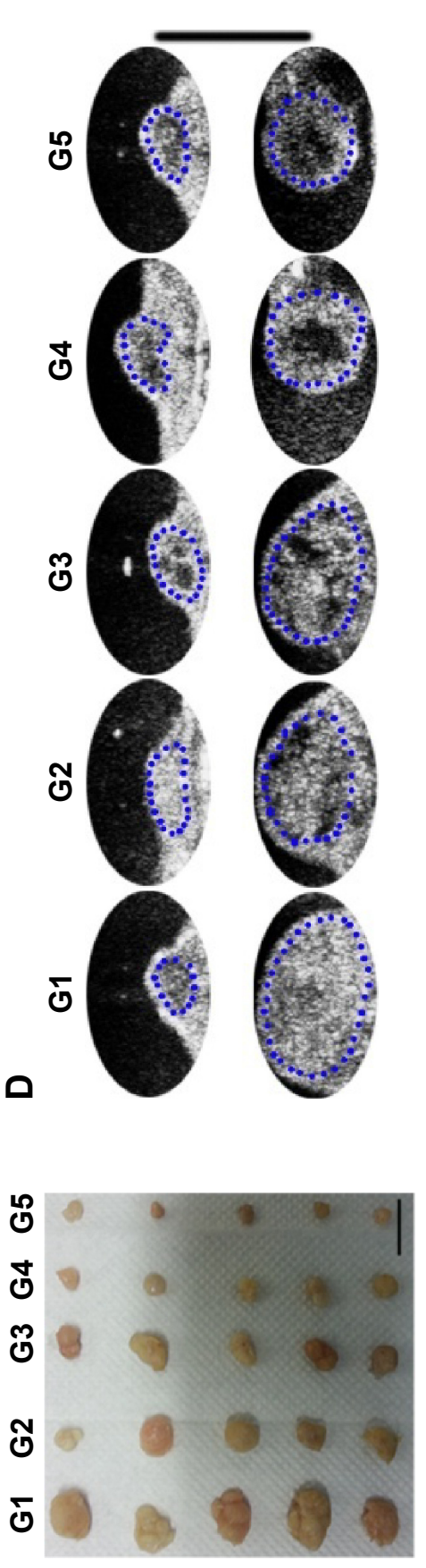

0

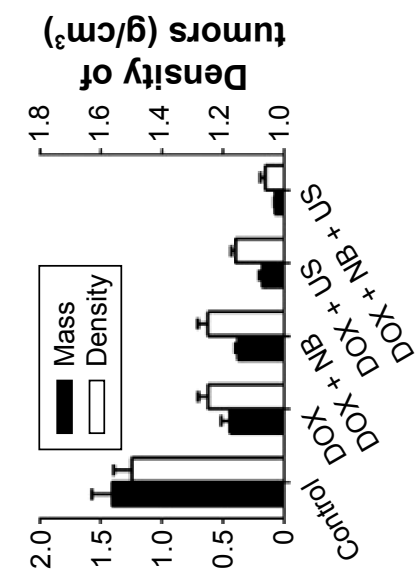

(6) sıoun?

I to ssew

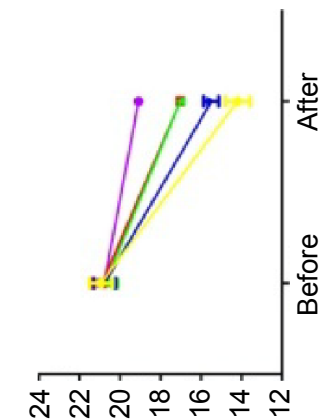

(ap) speub!s

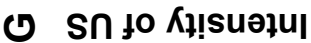

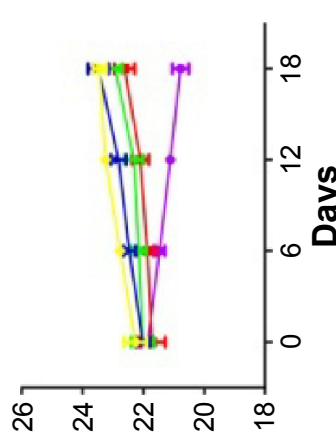

ᄂ (6) Ұчб! әм Кров

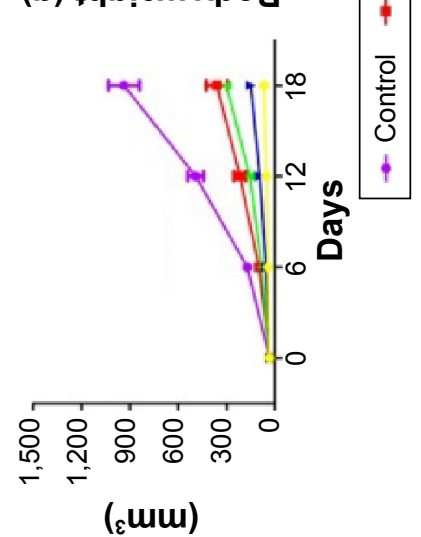

ш әunjOᄉ Joun $\perp$

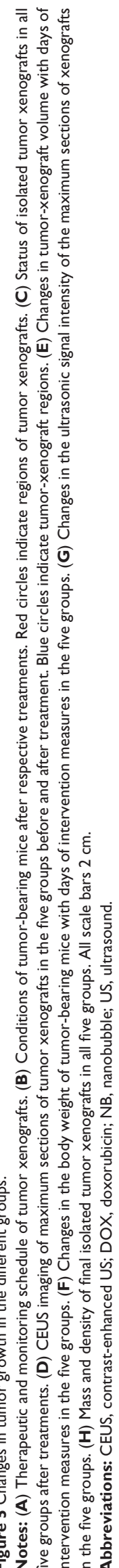



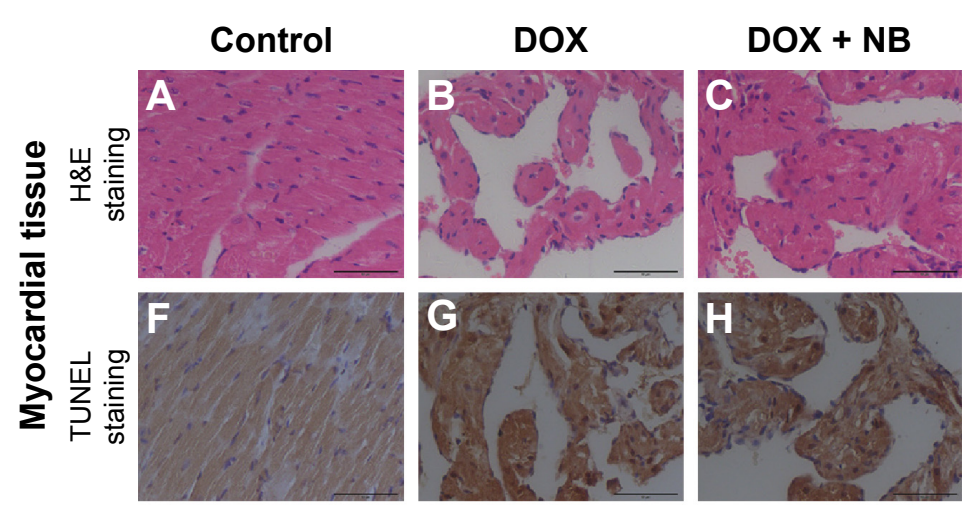

\section{DOX + US}
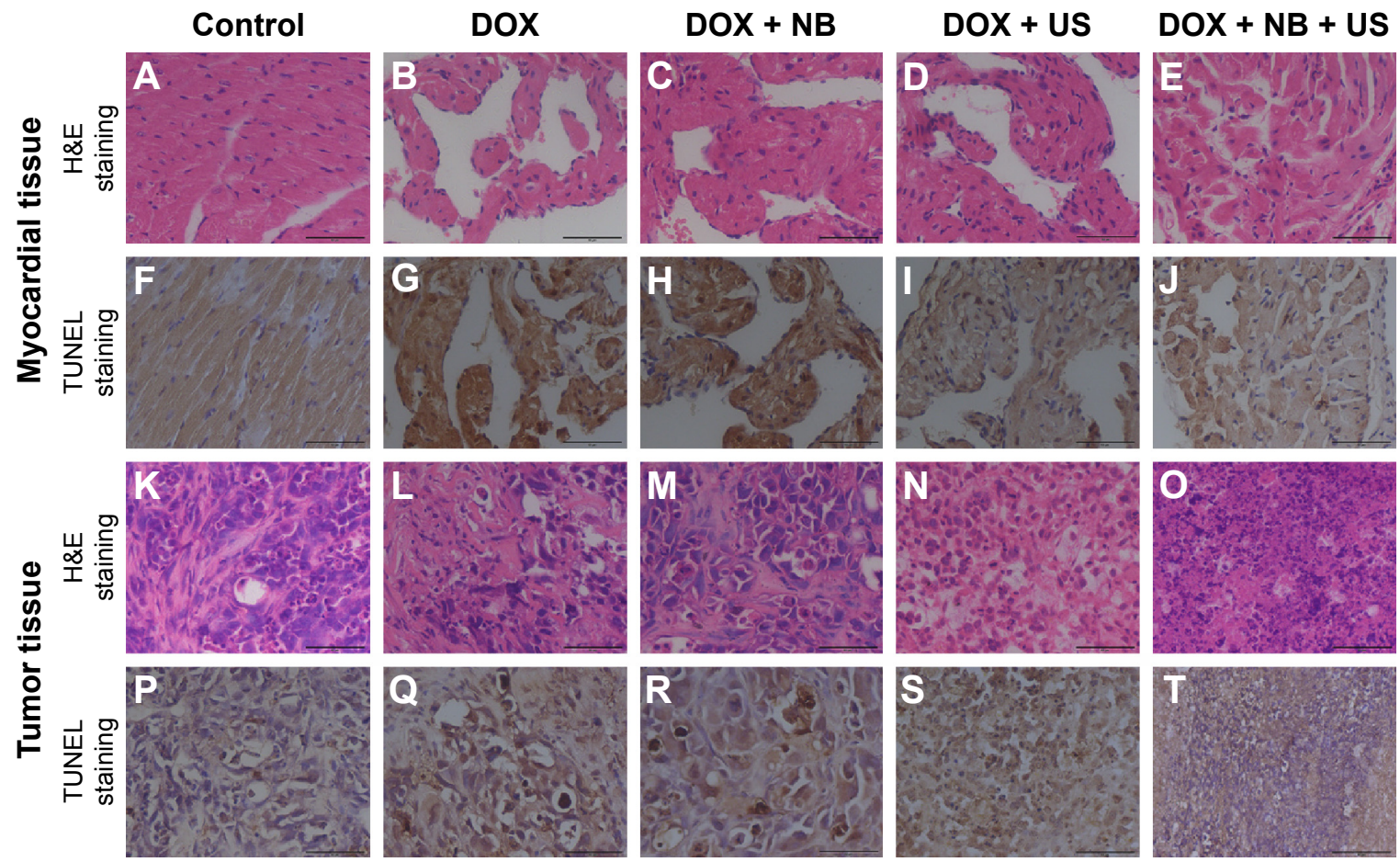

Figure 6 H\&E and TUNEL staining of myocardial tissues and xenograft tissues of nude mice in all groups.

Notes: (A-E) H\&E staining of myocardial tissues of all groups; (F-J) TUNEL staining of myocardial tissues of all groups; (K-O) H\&E staining of xenograft tissues of all groups; and (P-T) TUNEL staining of xenograft tissues of all groups. Scale bars $50 \mu \mathrm{m}$.

Abbreviations: DOX, doxorubicin; H\&E, hematoxylin-eosin; NB, nanobubble; TUNEL, terminal deoxynucleotidyl transferase deoxyuridine triphosphate nick end labeling; US, ultrasound.
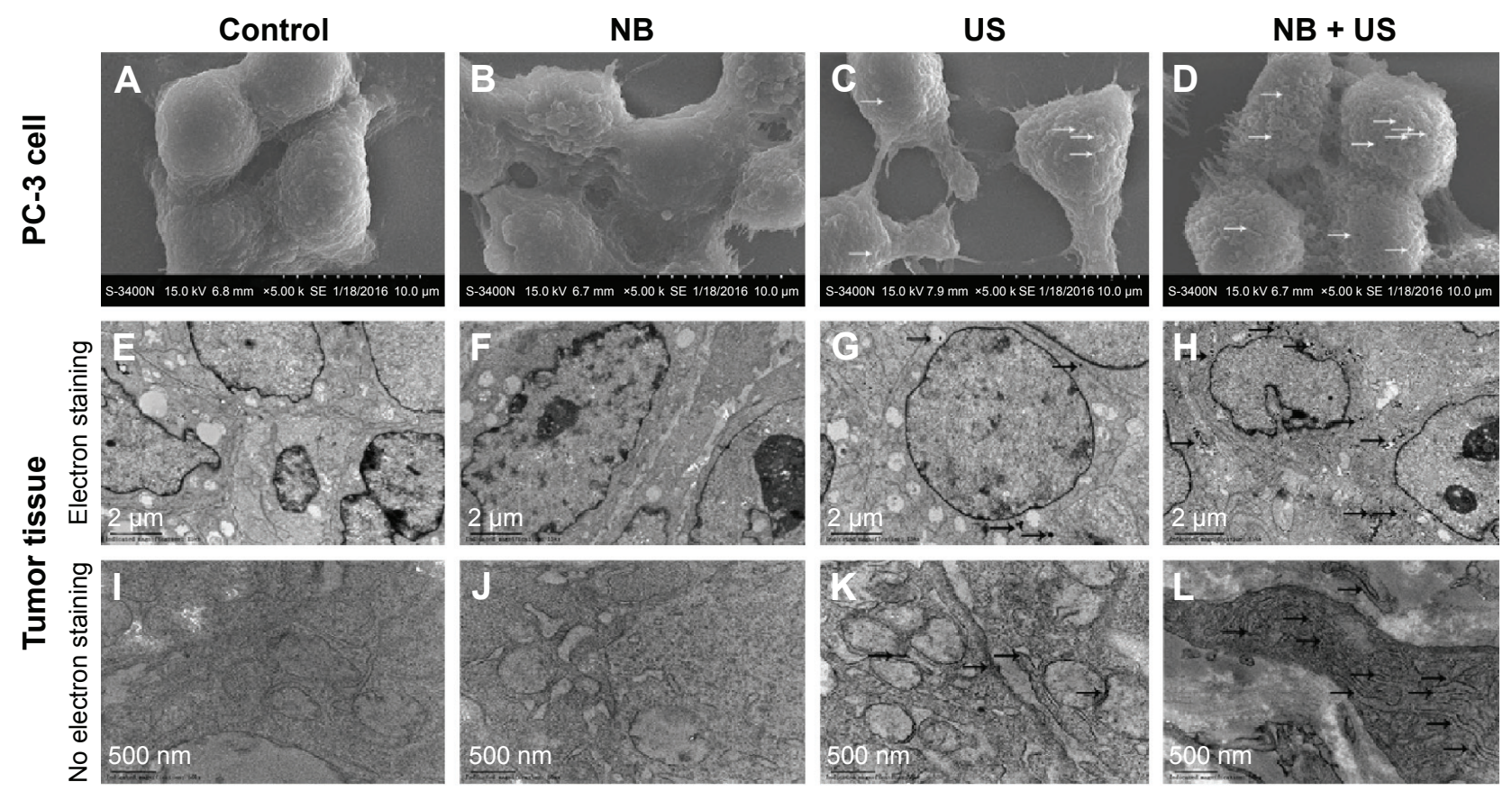

Figure 7 Cavitation effect of US burst of NBs on cells and tumor xenografts.

Notes: (A-D) Effects of the four types of treatment on the cell surface visualized under SEM. White arrows indicate pores on the cell surface. (E-H) Condition of lanthanum nitrate particles in tumor xenografts of all treatment groups visualized under TEM after electron staining. Black arrows indicate the locations of lanthanum nitrate-particle deposition. (I-L) Observation of the status of lanthanum nitrate particles in tumor xenografts under TEM without electron staining. Black arrows indicate the locations of lanthanum nitrate-particle deposition.

Abbreviations: NBs, nanobubbles; SEM, scanning electron microscopy; TEM, transmission electron microscopy; US, ultrasonic irradiation. 
distribution of lanthanum agents in tumor xenografts without electron staining was observed. The results showed that the NB + US group had the most extensive distribution of lanthanum agents, which were mostly observed in the mitochondria and endoplasmic reticulum. There were few lanthanum particles in the control and NB groups (Figure 7I-L).

\section{Discussion}

Prostate cancer is a malignant tumor that severely threatens the health of elderly men. Currently, there are many types of treatment methods, of which chemotherapy is an important option for patients with surgical contraindications and metastasis. ${ }^{13,14}$ Because DOX belongs to cellcycle-nonspecific chemotherapeutic drugs, it has killing effects in a variety of tumors. The mechanism of action of DOX mainly involves entering cells to interact with cellular genetic materials, such as DNA and RNA, to produce killing effects on tumor cells. However, DOX has significant side effects, including myelosuppression and cardiotoxicity; therefore, its clinical application has certain restrictions, especially for elderly patients or patients with poor cardiac function. In addition, because DOX has the fluorophore feature, it is easily detected and quantitated; therefore, it has been extensively applied in many studies of tumor treatment using drug carriers, such as gold particles, magnetic particles, and microbubbles. . $^{15,16}$

Some researchers have reported that pure low-frequency US could promote the uptake and distribution of liposomal DOX in prostate cancer xenografts, which might be associated with the production of cavitation nuclei by fluid in xenografts, such as blood under US excitation. ${ }^{17}$ Since Gramiak et al first discovered that microbubbles could enhance US images in 1968, UCAs have received extensive attention. ${ }^{18}$ Numerous studies have confirmed that UCAs can be used as not only diagnostic agents but also a type of effective carrier to achieve the targeted delivery of drugs under US irradiation. In UTMD technology, UCAs are used as cavitation nuclei, and begin to produce periodic contractions and expansions around the equilibrium radius to generate radiation pressure and microjets at surroundings under specific acoustic pressures, which is termed stable cavitation. With further increases in US intensity, UCAs are ruptured, and the generated high heat and high pressure can result in the production of strong shock waves, high-speed jet flows, and free radicals; this effect is termed inertial cavitation. Cavitation is an important basis of UTMD technology, and can increase tissue and vascular endothelial gaps and membrane permeability to increase the entry of drugs into parenchymal cells at the US-irradiation location to exert their functions.
Cavitation is typically applied in studies of the opening of the blood-brain barrier. ${ }^{19-21}$ A large number of studies have reported that UTMD technology promoted the release of many substances inside UCAs or carried by the outer shell, such as chemotherapeutic drugs and genes, to kill tumor cells at a short distance, thus effectively treating tumors. ${ }^{22-24}$

Other researchers have prepared UCAs using degradable polymer outer shells to encapsulate liquid droplets, which were injected into animals and entered tissue gaps through tumor endothelial gaps. These NBs coalesced into highly echogenic microbubbles to release encapsulated drugs under US irradiation. ${ }^{25}$ In the aforementioned studies on the production of cavitation by US irradiation combined with UCA, they were mainly at the micron level. Our previous studies confirmed that NBs could enter tumor-tissue gaps through the EPR effect of tumor tissues to exert important functions in the targeted diagnosis of tumors. However, further investigation is still required to determine whether cavitation can be produced or promoted under US irradiation to promote the function of drugs/genes on tumors at a short distance. Based on these considerations, we examined whether the features of lipid NBs, including their strong penetration ability and more extensive distribution in tumor tissues compared with microbubbles, could be combined with the traditional chemotherapeutic drug DOX to investigate their effects on prostate cancer and underlying mechanism under US irradiation. The results might provide a basis for further in-depth studies of the use of US NBs as a drug/gene-delivery system.

The preparation of a drug or gene carrying UCAs involves two main methods. One method is directly to adhere drugs or genes to the outer membrane of UCAs (adhesion method), and the other method is to consolidate drugs or genes inside the membrane (integration method). ${ }^{26}$ Although current UCAs have many different types of outer shells (including proteins, lipid materials, and polymers), UCAs with lipid shells have excellent acoustic features and safety; therefore, this type of UCA has the most extensive applications in clinical settings and laboratories. Studies of US therapy have shown that NBs composed mainly of polymers also have excellent functions in the encapsulation of drugs, for example, it has been reported that through the construction of polymer NBs, the encapsulation of a sufficient amount of DOX was achieved, which had significant inhibitory effects on tumors. ${ }^{27}$ However, physical conditions during their preparation are complex and difficult to control. In addition, the destruction of polymer UCAs requires high-intensity US-power output, which has damaging effects on tissues on the acoustic channel.

Although UCAs with lipid shells have excellent safety, the lipid shell is thin, and the particle size of UCAs is small, 
such that the amount of drug carried by the UCAs is limited. To achieve therapeutic effects, the amount of a lipid UCA is usually ten- to 100 -fold that in diagnosis, which not only greatly increases the cost but also has safety issues when applying large doses. Our prepared NBs also had the same issue. ${ }^{28}$ Therefore, to investigate the effects of NBs on the promotion of DOX to inhibit prostate cancer growth under US irradiation and the underlying mechanism, this study used the method of injecting a mixture of DOX and NBs to ensure the proper DOX concentration. The effects on the inhibition of prostate cancer cells and xenografts under UTND were studied, and the related mechanism was further analyzed. The results showed that the inhibition rate of cell growth and DOX concentration in xenografts were highest in the DOX + NB + US group, and gradually reduced in the order of the DOX + US, DOX + $\mathrm{NB}$, and DOX groups; however, the DOX concentrations in myocardial tissues showed the opposite trend. In addition, the reduction in xenograft volume after nine-time interventions was the most significant in the DOX + NB + US group. TUNEL staining results of myocardial tissues and tumor xenografts further showed that the xenograft tissues in the $\mathrm{DOX}+\mathrm{NB}+\mathrm{US}$ and DOX + US groups had cellular necrosis, which was most significant in the former group. Myocardial tissues had only a few apoptotic cells, indicating that NBs could increase the killing effects of DOX in irradiated regions (xenograft location) under UTND technology.

Under the condition that the total amount of injected DOX was the same, UTND could change the distribution of DOX and reduce the distribution of DOX in other unirradiated tissues and organs, thus reducing the toxic effects of DOX on the heart. SEM results showed that there were more holes in prostate cancer cells in the DOX + NB + US group, and cell-surface folds were significantly increased. In the observation of tumor xenografts using TEM, lanthanum nitrate with a molecular weight of 433 and a mean particle size of $\sim 40 \AA$ was used as an electron tracer of ultrastructure (when the permeability of the cell membrane was normal, lanthanum nitrate was present only in extracellular gaps and could not enter cells). The results showed that UTND could increase the uptake of lanthanum nitrate by tumor xenografts, indicating that the UTND technology was conducive to increasing the permeability of tumor-cell membranes. This method was effective for promoting drugs to act on specific sites, and could effectively increase the concentration of drugs in tumor regions. The increase in holes on the cell surface and the increase in permeability both confirmed that UTND technology could produce cavitation effects and provides evidence for relevant studies on the promotion of tumor treatment by genes or drugs.
The injection method for the mixture of drug and NBs used in this study had the following features, which are worthy of further in-depth studies and clinical translation: 1) Because lipid microbubbles have been extensively applied in the clinic, NBs using lipids as the outer shell have excellent safety features in human applications; 2) although DOX and NBs were injected into the vein as a mixture, our results confirmed that NBs altered the drug distribution under US irradiation to reduce the toxic effects of DOX on the heart, which effectively reduced damaging effects on other normal tissues at the same time as increased treatment effects compared to using DOX alone; 3) the analysis of the related mechanism showed that US irradiation on NBs produced cavitation effects similar to microbubbles to promote the permeability of tumor cells, which would be helpful for drugs or genes to produce tumor-killing effects in specific regions of US irradiation; 4) we selected $1 \mathrm{~W} /$ $\mathrm{cm}^{2}$ US power in this experiment to perform 15 minutes of $100 \mathrm{~Hz}$ combined with the 5 seconds on-5 seconds off irradiation mode on tumor regions. On the basis of ensuring full perfusion and burst of all the NBs in the body, the thermal effects of the therapeutic US were reduced to the greatest extent possible, which was conducive for treatment targeting internal solid tumors and did not damage normal tissues on the acoustic channel at the same time. Therefore, the distribution of cytotoxic drugs to tumors through the circulation is increased, and nontarget-organ damage is reduced. ${ }^{29}$

However, our study also had the following limitations. Although the low-intensity US therapeutic equipment used in this study can be easily and rapidly adjusted, the available parameters were limited and the irradiation time was controlled manually, together with built-in parameters in the apparatus. Also, we did not include measurements of such parameters as cavitation-threshold values or resonance frequency of our fabricated lipid NBs, which should be resolved in further study.

\section{Conclusion}

This study confirmed that UTND can produce cavitation effects to promote the effects of DOX on the inhibition of prostate cancer growth. In addition, under a specific drug concentration in the body, this technique can change the distribution of drugs in the body to reduce toxic side effects on normal tissues. This study not only provides experimental methods and evidence for the delivery of chemotherapeutic drugs by NBs under a low-intensity US burst but also lays the foundation for future relevant studies on tumor treatment using NBs as drug/gene-delivery carriers. 


\section{Acknowledgments}

This work was supported by the International Science and Technology Cooperation Program of China (2015DFA30920), the National Natural Science Foundation of China (81571732), and the Science and Technology (International Science and Technology Cooperation) Research Base Construction Program of Chongqing (cstc2014gjhz110004).

\section{Disclosure}

The authors report no conflicts of interest in this work.

\section{References}

1. Mayer CR, Bekeredjian R. Ultrasonic gene and drug delivery to the cardiovascular system. Adv Drug Deliv Rev. 2008;60(10):1177-1192.

2. Omata D, Negishi Y, Suzuki R, Oda Y, Endo-Takahashi Y, Maruyama K. Nonviral gene delivery systems by the combination of bubble liposomes and ultrasound. Adv Genet. 2015;89:25-48.

3. Zhang Y, Chang S, Sun J, et al. Targeted microbubbles for ultrasound mediated short hairpin RNA plasmid transfection to inhibit survivin gene expression and induce apoptosis of ovarian cancer A2780/DDP cells. Mol Pharm. 2015;12(9):3137-3145.

4. Zen K, Okigaki M, Hosokawa Y, et al. Myocardium-targeted delivery of endothelial progenitor cells by ultrasound-mediated microbubble destruction improves cardiac function via an angiogenic response. J Mol Cell Cardiol. 2006;40(6):799-809.

5. Yoshida J, Ohmori K, Takeuchi H, et al. Treatment of ischemic limbs based on local recruitment of vascular endothelial growth factorproducing inflammatory cells with ultrasonic microbubble destruction. J Am Coll Cardiol. 2005;46(5):899-905.

6. Xie W, Liu S, Su H, Wang Z, Zheng Y, Fu Y. Ultrasound microbubbles enhance recombinant adeno-associated virus vector delivery to retinal ganglion cells in vivo. Acad Radiol. 2010;17(10):1242-1248.

7. Zhou S, Li S, Liu Z, et al. Ultrasound-targeted microbubble destruction mediated herpes simplex virus-thymidine kinase gene treats hepatoma in mice. J Exp Clin Cancer Res. 2010;29:170.

8. Xing Z, Wang J, Ke H, et al. The fabrication of novel nanobubble ultrasound contrast agent for potential tumor imaging. Nanotechnology. 2010; 21(14): 145607.

9. Wang Y, Li X, Zhou Y, Huang P, Xu Y. Preparation of nanobubbles for ultrasound imaging and intracellular drug delivery. Int J Pharm. 2010; 384(1-2):148-153.

10. Perera RH, Hernandez C, Zhou H, Kota P, Burke A, Exner AA. Ultrasound imaging beyond the vasculature with new generation contrast agents. Wiley Interdiscip Rev Nanomed Nanobiotechnol. 2015;7(4):593-608.

11. Wang L, Zhang M, Tan K, et al. Preparation of nanobubbles carrying androgen receptor siRNA and their inhibitory effects on androgenindependent prostate cancer when combined with ultrasonic irradiation. PLoS One. 2014;9(5):e96586.

12. Tong HP, Wang LF, Guo YL, et al. Preparation of protamine cationic nanobubbles and experimental study of their physical properties and in vivo contrast enhancement. Ultrasound Med Biol. 2013;39(11): $2147-2157$.

International Journal of Nanomedicine

\section{Publish your work in this journal}

The International Journal of Nanomedicine is an international, peerreviewed journal focusing on the application of nanotechnology in diagnostics, therapeutics, and drug delivery systems throughout the biomedical field. This journal is indexed on PubMed Central, MedLine, CAS, SciSearch ${ }^{\circledR}$, Current Contents ${ }^{\circledR} /$ Clinical Medicine,
13. Siegel RL, Miller KD, Jemal A. Cancer statistics, 2015. CA Cancer J Clin. 2015;65(1):5-29.

14. Thalgott M, Horn T, Heck MM, et al. Long-term results of a phase II study with neoadjuvant docetaxel chemotherapy and complete androgen blockade in locally advanced and high-risk prostate cancer. J Hematol Oncol. 2014;7:20.

15. Tsai LC, Hsieh HY, Lu KY, Wang SY, Mi FL. EGCG/gelatin-doxorubicin gold nanoparticles enhance therapeutic efficacy of doxorubicin for prostate cancer treatment. Nanomedicine (Lond). 2016;11(1):9-30.

16. Zhang W, Zheng X, Shen S, Wang X. Doxorubicin-loaded magnetic nanoparticle clusters for chemo-photothermal treatment of the prostate cancer cell line PC3. Biochem Biophys Res Commun. 2015; 466(2):278-282.

17. Eggen S, Afadzi M, Nilssen EA, Haugstad SB, Angelsen B, Davies CL. Ultrasound improves the uptake and distribution of liposomal doxorubicin in prostate cancer xenografts. Ultrasound Med Biol. 2013; 39(7):1255-1266.

18. Gramiak R, Shah PM. Echocardiography of the aortic root. Invest Radiol. 1968;3(5):356-366.

19. Frulio N, Trillaud H, Deckers R, Lepreux S, Moonen C, Quesson B. Influence of ultrasound induced cavitation on magnetic resonance imaging contrast in the rat liver in the presence of macromolecular contrast agent. Invest Radiol. 2010;45(5):282-287.

20. Mayer CR, Geis NA, Katus HA, Bekeredjian R. Ultrasound targeted microbubble destruction for drug and gene delivery. Expert Opin Drug Deliv. 2008;5(10):1121-1138.

21. Bekeredjian R, Kuecherer HF, Kroll RD, Katus HA, Hardt SE. Ultrasound-targeted microbubble destruction augments protein delivery into testes. Urology. 2007;69(2):386-389.

22. Chang S, Si T, Zhang S, Merrick MA, Cohn DE, Xu RX. Ultrasound mediated destruction of multifunctional microbubbles for image guided delivery of oxygen and drugs. Ultrason Sonochem. 2016;28:31-38.

23. Cavalli R, Bisazza A, Giustetto P, et al. Preparation and characterization of dextran nanobubbles for oxygen delivery. Int J Pharm. 2009;381(2): $160-165$.

24. Geis NA, Katus HA, Bekeredjian R. Microbubbles as a vehicle for gene and drug delivery: current clinical implications and future perspectives. Curr Pharm Des. 2012;18(15):2166-2183.

25. Gao Z, Kennedy AM, Christensen DA, Rapoport NY. Drug-loaded nano/microbubbles for combining ultrasonography and targeted chemotherapy. Ultrasonics. 2008;48(4):260-270.

26. Xu Y, Xie Z, Zhou Y, et al. Experimental endostatin-GFP gene transfection into human retinal vascular endothelial cells using ultrasound-targeted cationic microbubble destruction. Mol Vis. 2015;21:930-938.

27. Meng M, Gao J, Wu C, et al. Doxorubicin nanobubble for combining ultrasonography and targeted chemotherapy of rabbit with VX2 liver tumor. Tumour Biol. Epub 2016 Jan 6.

28. Li P, Zheng Y, Ran H, et al. Ultrasound triggered drug release from 10-hydroxycamptothecin-loaded phospholipid microbubbles for targeted tumor therapy in mice. J Control Release. 2012;162(2): 349-354.

29. Fan X, Wang L, Guo Y, et al. Experimental investigation of the penetration of ultrasound nanobubbles in a gastric cancer xenograft. Nanotechnology. 2013;24(32):325102.

Journal Citation Reports/Science Edition, EMBase, Scopus and the Elsevier Bibliographic databases. The manuscript management system is completely online and includes a very quick and fair peer-review system, which is all easy to use. Visit http://www.dovepress.com/ testimonials.php to read real quotes from published authors. 